\title{
ANALYTIC SOLUTION OF CERTAIN SECOND-ORDER FUNCTIONAL DIFFERENTIAL EQUATION
}

\author{
THEERADACH KAEWONG AND PIYAPONG NIAMSUP
}

Received 28 September 2005; Revised 2 July 2006; Accepted 9 July 2006

We consider the existence of analytic solutions of a certain class of iterative second-order functional differential equation of the form $x^{\prime \prime}\left(x^{[r]}(z)\right)=c_{0} z^{2}+c_{1}(x(z))^{2}+\left(c_{2} x^{[2]}(z)\right)^{2}+$ $\cdots+c_{m}\left(x^{[m]}(z)\right)^{2}, m, r \geq 0$.

Copyright @ 2006 Hindawi Publishing Corporation. All rights reserved.

\section{Introduction}

In recent years, the study of the existence of analytic solutions of iterative functional differential equations has attracted several researchers, see [2-11] and references cited therein. In [3], the authors studied the existence of analytic solutions of iterative functional differential equation of the following form:

$$
x^{\prime \prime}(z)=\left(x^{[m]}(z)\right)^{2}
$$

where $m$ is a nonnegative integer. In the present paper, we propose to study a more general form of iterative functional differential equations than (1.1) as follows:

$$
x^{\prime \prime}\left(x^{[r]}(z)\right)=c_{0} z^{2}+c_{1}(x(z))^{2}+c_{2}\left(x^{[2]}(z)\right)^{2}+\cdots+c_{m}\left(x^{[m]}(z)\right)^{2},
$$

where $r$ and $m$ are nonnegative integers, $c_{0}, c_{1}, c_{2}, \ldots, c_{m}$ are complex numbers, $\sum_{j=0}^{m}\left|c_{j}\right| \neq$ 0 , and $x^{[j]}$ denotes the $j$ th iterate of $x$. In order to obtain analytic solutions of (1.1), we first seek the analytic solutions $y(z)$ of the following companion equation:

$$
\alpha^{2} y^{\prime \prime}\left(\alpha^{r+1} z\right) y^{\prime}\left(\alpha^{r} z\right)=\alpha y^{\prime}\left(\alpha^{r+1} z\right) y^{\prime \prime}\left(\alpha^{r} z\right)+\left[y^{\prime}\left(\alpha^{r} z\right)\right]^{3}\left[\sum_{j=0}^{m} c_{j}\left(y\left(\alpha^{j} z\right)\right)^{2}\right]
$$

satisfying the initial value conditions

$$
y(0)=\mu, \quad y^{\prime}(0)=\eta \neq 0,
$$

Hindawi Publishing Corporation

International Journal of Mathematics and Mathematical Sciences

Volume 2006, Article ID 53828, Pages 1-16

DOI 10.1155/IJMMS/2006/53828 
2 Analytic solution of functional differential equation

where $\mu, \eta$ are complex numbers, and $\alpha$ satisfies one of the following conditions:

(H1) $|\alpha|>1$;

(H2) $0<|\alpha|<1$;

(H3) $|\alpha|=1, \alpha$ is not a root of unity, and $\log \left(1 /\left|\alpha^{n}-1\right|\right) \leq K \log n, n=2,3,4, \ldots$,

for some positive constant $K$. Then we show that (1.2) has an analytic solution of the form

$$
x(z)=y\left(\alpha y^{-1}(z)\right)
$$

in a neighborhood of the number $\mu$, where $y^{-1}(z)$ is the inverse function of $y(z)$. Finally, we make use of (1.5) to show how to derive an explicit power series solution of (1.2).

\section{Preliminary lemmas}

We first obtain the analytic solutions $y(z)$ of the companion equation (1.3). By (1.3), we have that

$$
\begin{gathered}
\frac{\alpha^{2} y^{\prime \prime}\left(\alpha^{r+1} z\right) y^{\prime}\left(\alpha^{r} z\right)-\alpha y^{\prime}\left(\alpha^{r+1} z\right) y^{\prime \prime}\left(\alpha^{r} z\right)}{\left[y^{\prime}\left(\alpha^{r} z\right)\right]^{2}}=y^{\prime}\left(\alpha^{r} z\right) \sum_{j=0}^{m} c_{j}\left(y\left(\alpha^{j} z\right)\right)^{2}, \quad \text { or } \\
\frac{1}{\alpha^{r-1}}\left(\frac{y^{\prime}\left(\alpha^{r+1} z\right)}{y^{\prime}\left(\alpha^{r} z\right)}\right)^{\prime}=y^{\prime}\left(\alpha^{r} z\right) \sum_{j=0}^{m} c_{j}\left(y\left(\alpha^{j} z\right)\right)^{2}, \quad \text { or } \\
\frac{1}{\alpha^{r-1}}\left[\frac{y^{\prime}\left(\alpha^{r+1} z\right)}{y^{\prime}\left(\alpha^{r} z\right)}-\frac{y^{\prime}(0)}{y^{\prime}(0)}\right]=\int_{0}^{z} y^{\prime}\left(\alpha^{r} t\right) \sum_{j=0}^{m} c_{j}\left(y\left(\alpha^{j} t\right)\right)^{2} d t, \quad \text { or } \\
\frac{1}{\alpha^{r-1}}\left[\frac{y^{\prime}\left(\alpha^{r+1} z\right)}{y^{\prime}\left(\alpha^{r} z\right)}-1\right]=\int_{0}^{z} y^{\prime}\left(\alpha^{r} t\right) \sum_{j=0}^{m} c_{j}\left(y\left(\alpha^{j} t\right)\right)^{2} d t, \quad \text { or } \\
\frac{1}{\alpha^{r-1}}\left[y^{\prime}\left(\alpha^{r+1} z\right)-y^{\prime}\left(\alpha^{r} z\right)\right]=y^{\prime}\left(\alpha^{r} z\right) \int_{0}^{z} y^{\prime}\left(\alpha^{r} t\right) \sum_{j=0}^{m} c_{j}\left(y\left(\alpha^{j} t\right)\right)^{2} d t .
\end{gathered}
$$

Since $y(z)$ is an analytic function in a neighborhood of $0, y(z)$ can be represented by a power series of the form

$$
y(z)=\sum_{n=0}^{+\infty} b_{n} z^{n},
$$

and we can see easily that $b_{0}=\mu, b_{1}=\eta$, and $y^{\prime}(z)=\sum_{n=0}^{+\infty}(n+1) b_{n+1} z^{n}$. We have

$$
\begin{aligned}
\frac{1}{\alpha^{r-1}} & {\left[y^{\prime}\left(\alpha^{r+1} z\right)-y^{\prime}\left(\alpha^{r} z\right)\right] } \\
& =\frac{1}{\alpha^{r-1}}\left[\sum_{n=0}^{+\infty}(n+1) b_{n+1} \alpha^{(r+1) n} z^{n}-\sum_{n=0}^{+\infty}(n+1) b_{n+1} \alpha^{r n} z^{n}\right] \\
& =\frac{1}{\alpha^{r-1}}\left[\sum_{n=0}^{+\infty}(n+1) b_{n+1}\left(\alpha^{n}-1\right) \alpha^{r n} z^{n}\right]
\end{aligned}
$$




$$
\begin{aligned}
& =\frac{1}{\alpha^{r-1}}\left[\sum_{n=1}^{+\infty}(n+1) b_{n+1}\left(\alpha^{n}-1\right) \alpha^{r n} z^{n}\right] \\
& =\frac{1}{\alpha^{r-1}}\left[\sum_{n=0}^{+\infty}(n+2) b_{n+2}\left(\alpha^{n+1}-1\right) \alpha^{r(n+1)} z^{n+1}\right] \\
& =\sum_{n=0}^{+\infty}(n+2)\left(\alpha^{n+1}-1\right) \alpha^{r n+1} b_{n+2} z^{n+1} .
\end{aligned}
$$

Therefore,

$$
\frac{1}{\alpha^{r-1}}\left[y^{\prime}\left(\alpha^{r+1} z\right)-y^{\prime}\left(\alpha^{r} z\right)\right]=\sum_{n=0}^{+\infty}(n+2)\left(\alpha^{n+1}-1\right) \alpha^{r n+1} b_{n+2} z^{n+1} .
$$

By means of (2.2), we get that

$$
y^{2}(z)=\left(\sum_{n=0}^{+\infty} b_{n} z^{n}\right)^{2}=\sum_{n=0}^{+\infty}\left(\sum_{i=0}^{n} b_{i} b_{n-i}\right) z^{n} .
$$

Then

$$
y^{2}\left(\alpha^{j} z\right)=\sum_{n=0}^{+\infty}\left(\sum_{i=0}^{n} b_{i} b_{n-i}\right) \alpha^{j n} z^{n}, \quad j=0,1,2, \ldots, m
$$

This implies

$$
\begin{aligned}
\int_{0}^{z} y^{\prime}\left(\alpha^{r} t\right) \sum_{j=0}^{m} c_{j}\left(y\left(\alpha^{j} t\right)\right)^{2} d t \\
=\int_{0}^{z}\left(\sum_{n=0}^{+\infty}(n+1) b_{n+1} \alpha^{r n} t^{n}\right)\left(\sum_{n=0}^{+\infty} \sum_{i=0}^{n} \sum_{j=0}^{m} c_{j} \alpha^{j n} b_{i} b_{n-i} t^{n}\right) d t \\
=\int_{0}^{z} \sum_{n=0}^{+\infty} \sum_{k=0}^{n} \sum_{i=0}^{n-k} \sum_{j=0}^{m} c_{j} \alpha^{(n-k) j+r}(k+1) b_{i} b_{k+1} b_{n-k-i} t^{n} d t \\
=\sum_{n=0}^{+\infty}\left(\frac{\sum_{k=0}^{n} \sum_{i=0}^{n-k} \sum_{j=0}^{m} c_{j} \alpha^{(n-k) j+k r}}{n+1}(k+1) b_{i} b_{k+1} b_{n-k-i}\right) z^{n+1} .
\end{aligned}
$$

Therefore,

$$
\int_{0}^{z} y^{\prime}\left(\alpha^{r} t\right) \sum_{j=0}^{m} c_{j}\left(y\left(\alpha^{j} t\right)\right)^{2} d t=\sum_{n=0}^{+\infty} \frac{\sum_{k=0}^{n} \sum_{i=0}^{n-k} \sum_{j=0}^{m} c_{j} \alpha^{(n-k) j+(k+1) r}}{n+1}(k+1) b_{i} b_{k+1} b_{n-k-i} z^{n+1} .
$$


4 Analytic solution of functional differential equation

Next, we will consider

$$
\begin{aligned}
y^{\prime}\left(\alpha^{r} z\right) & \int_{0}^{z} y^{\prime}\left(\alpha^{r} t\right) \sum_{j=0}^{m} c_{j}\left(y\left(\alpha^{j} t\right)\right)^{2} d t \\
= & \left(\sum_{n=0}^{+\infty}(n+1) b_{n+1} \alpha^{r n} z^{n}\right) \sum_{n=0}^{+\infty} \frac{\sum_{k=0}^{n} \sum_{i=0}^{n-k} \sum_{j=0}^{m} c_{j} \alpha^{(n-k) j+k r}}{n+1}(k+1) b_{i} b_{k+1} b_{n-k-i} z^{n+1} \\
= & \sum_{n=0}^{+\infty} \frac{\sum_{s=0}^{n} \sum_{k=0}^{n-s} \sum_{i=0}^{n-k-s} \sum_{j=0}^{m} c_{j} \alpha^{(n-s-k) j+(k+s) r}}{n-s+1}(s+1)(k+1) b_{i} b_{s+1} b_{k+1} b_{n-s-k-i} z^{n+1} .
\end{aligned}
$$

Therefore,

$$
\begin{aligned}
& y^{\prime}\left(\alpha^{r} z\right) \int_{0}^{z} y^{\prime}\left(\alpha^{r} t\right) \sum_{j=0}^{m} c_{j}\left(y\left(\alpha^{j} t\right)\right)^{2} d t \\
& \quad=\sum_{n=0}^{+\infty} \frac{\sum_{s=0}^{n} \sum_{k=0}^{n-s} \sum_{i=0}^{n-k-s} \sum_{j=0}^{m} c_{j} \alpha^{(n-s-k) j+(k+s) r}}{n-s+1}(s+1)(k+1) b_{i} b_{s+1} b_{k+1} b_{n-s-k-i} z^{n+1}
\end{aligned}
$$

We see that (1.3) is equivalent to the integrodifferential equation (2.1). By (2.1), (2.4), and (2.10), we see that

$$
\begin{aligned}
(n+2)\left(\alpha^{n+1}-1\right) \alpha^{r n+1} b_{n+2}= & \frac{\sum_{s=0}^{n} \sum_{k=0}^{n-s} \sum_{i=0}^{n-k-s} \sum_{j=0}^{m} c_{j} \alpha^{(n-s-k) j+(k+s) r}}{n-s+1} \\
& \times(s+1)(k+1) b_{i} b_{s+1} b_{k+1} b_{n-s-k-i}, \quad n=0,1,2 \ldots
\end{aligned}
$$

Therefore,

$$
b_{n+2}=\frac{\sum_{s=0}^{n} \sum_{k=0}^{n-s} \sum_{i=0}^{n-k-s} \sum_{j=0}^{m} c_{j} \alpha^{(n-s-k) j+(k+s) r-r n-1}}{(n+2)(n-s+1)\left(\alpha^{n+1}-1\right)}(s+1)(k+1) b_{i} b_{s+1} b_{k+1} b_{n-s-k-i},
$$

where $n=0,1,2 \ldots$ Next, we show that such a power series solution is majorized by a convergent power series. Now we begin with the following preparatory lemma, the proof of which can be found in [1, Chapter 6].

Lemma 2.1. Assume that (H3) holds. Then there is a positive number $\delta$ such that $\mid \alpha^{n}-$ $\left.1\right|^{-1}<(2 n)^{\delta}$ for $n=1,2,3, \ldots$. Furthermore, the sequence $\left\{d_{n}\right\}_{n=1}^{\infty}$ defined by $d_{1}=1$ and $d_{n}=\left(1 /\left|\alpha^{n-1}-1\right|\right) \max _{n=n_{1}+n_{2}+\cdots+n_{t}, 0<n_{1} \leq n_{2} \leq \cdots \leq n_{t}, t \geq 2}\left\{d_{n_{1}} d_{n_{2}} \cdots d_{n_{t}}\right\}, n=2,3,4, \ldots$ satisfy $d_{n} \leq\left(2^{5 \delta+1}\right)^{n-1} n^{-2 \delta}, n=1,2,3, \ldots$

Lemma 2.2. Suppose that (H3) holds. Then, when $0<|\eta| \leq 1,(1.3)$ has an analytic solution of the form (2.2) in a neighborhood of the origin. 
Proof. For convenience, we let $M=\sum_{j=0}^{m}\left|c_{j}\right|$. By means of (2.12), it follows that for each $n=0,1,2, \ldots$,

$$
\begin{aligned}
\left|b_{n+2}\right| \leq & \frac{\sum_{s=0}^{n} \sum_{k=0}^{n-s} \sum_{i=0}^{n-k-s} \sum_{j=0}^{m}\left|c_{j}\right||\alpha|^{(n-s-k) j+(k+s) r-r n-1}}{(n+2)(n-s+1)\left|\left(\alpha^{n+1}-1\right)\right|} \\
& \times(s+1)(k+1)\left|b_{i}\right|\left|b_{s+1}\right|\left|b_{k+1}\right|\left|b_{n-s-k-i}\right| \\
\leq & \frac{M}{\left|\left(\alpha^{n+1}-1\right)\right|} \sum_{s=0}^{n} \sum_{k=0}^{n-s} \sum_{i=0}^{n-k-s}\left|b_{i}\right|\left|b_{s+1}\right|\left|b_{k+1}\right|\left|b_{n-s-k-i}\right| .
\end{aligned}
$$

Therefore,

$$
\left|b_{n+2}\right| \leq \frac{M}{\left|\left(\alpha^{n+1}-1\right)\right|} \sum_{s=0}^{n} \sum_{k=0}^{n-s} \sum_{i=0}^{n-k-s}\left|b_{i}\right|\left|b_{s+1}\right|\left|b_{k+1}\right|\left|b_{n-s-k-i}\right|,
$$

where $n=0,1, \ldots$ Let

$$
Q(z, \omega)=\omega^{4}-2|\mu| \omega^{3}+|\mu|^{2} \omega^{2}-\frac{1}{M}(\omega-|\mu|-z)
$$

for $(z, \omega)$ in a neighbor of $(0,|\mu|)$. We see that $Q(0,|\mu|)=|\mu|^{4}-2|\mu|^{4}+|\mu|^{4}-(1 / M)(|\mu|-$ $|\mu|-0)=0$ and $Q_{\omega}^{\prime}(z, \omega)=4 \omega^{3}-6|\mu| \omega^{2}+2|\mu|^{2} \omega-1 / M$, so $Q_{\omega}^{\prime}(0,|\mu|)=-1 / M \neq 0$. Therefore, there exists a unique analytic function $G(z)$ in a neighborhood of 0 such that $G(0)=|\mu|, G^{\prime}(0)=1$ satisfy the equality $Q(z, G(z))=0$. It follows that

$$
G(z)=\sum_{n=0}^{+\infty} C_{n} z^{n}
$$

where $C_{0}=|\mu|, C_{1}=1$ in a neighborhood of 0 . Next, we will show that

$$
C_{n+2}=M \sum_{s=0}^{n} \sum_{k=0}^{n-s} \sum_{i=0}^{n-k-s} C_{i} C_{s+1} C_{k+1} C_{n-s-k-i}, \quad n=0,1, \ldots
$$

Suppose that (2.17) is true, by (2.16), we will get that

$$
\begin{aligned}
G^{3}(z)= & G(z) G^{2}(z)=\left(C_{0}+\sum_{n=0}^{+\infty} C_{n+1} z^{n+1}\right)\left(\sum_{n=0}^{+\infty}\left(\sum_{i=0}^{n} C_{i} C_{n-i}\right) z^{n}\right) \\
= & C_{0} \sum_{n=0}^{+\infty}\left(\sum_{i=0}^{n} C_{i} C_{n-i}\right) z^{n}+\sum_{n=0}^{+\infty}\left(\sum_{k=0}^{n} \sum_{i=0}^{n-k} C_{i} C_{k+1} C_{n-k-i}\right) z^{n+1}, \\
G^{4}(z)= & G(z) G^{3}(z)=\left(C_{0}+\sum_{n=0}^{+\infty} C_{n+1} z^{n+1}\right) \\
& \times\left[C_{0} \sum_{n=0}^{+\infty}\left(\sum_{i=0}^{n} C_{i} C_{n-i}\right) z^{n}+\sum_{n=0}^{+\infty}\left(\sum_{k=0}^{n} \sum_{i=0}^{n-k} C_{i} C_{k+1} C_{n-k-i}\right) z^{n+1}\right]
\end{aligned}
$$


Analytic solution of functional differential equation

$$
\begin{aligned}
= & C_{0}^{2} \sum_{n=0}^{+\infty}\left(\sum_{i=0}^{n} C_{i} C_{n-i}\right) z^{n}+2 C_{0} \sum_{n=0}^{+\infty}\left(\sum_{k=0}^{n} \sum_{i=0}^{n-k} C_{i} C_{k+1} C_{n-k-i}\right) z^{n+1} \\
& +\sum_{n=0}^{+\infty}\left(M \sum_{s=0}^{n} \sum_{k=0}^{n-s} \sum_{i=0}^{n-k-s} C_{i} C_{s+1} C_{k+1} C_{n-s-k-i}\right) z^{n+2} \\
= & C_{0}^{2} G^{2}(z)+2 C_{0}\left[G^{3}(z)-C_{0} G^{2}(z)\right]+\frac{1}{M} \sum_{n=0}^{+\infty} C_{n+2} z^{n+2} \\
= & 2 C_{0} G^{3}(z)-C_{0}^{2} G^{2}(z)+\frac{1}{M}\left(G(z)-C_{0}-C_{1} z\right) \\
= & 2|\mu| G^{3}(z)-|\mu|^{2} G^{2}(z)+\frac{1}{M}(G(z)-|\mu|-z),
\end{aligned}
$$

that is,

$$
G^{4}(z)=2|\mu| G^{3}(z)-|\mu|^{2} G^{2}(z)+\frac{1}{M}(G(z)-|\mu|-z),
$$

or

$$
G^{4}(z)-2|\mu| G^{3}(z)+|\mu|^{2} G^{2}(z)-\frac{1}{M}(G(z)-|\mu|-z)=0 .
$$

Hence, $Q(z, G(z))=0$. Furthermore, we see that $Q(z, G(z))=0$ if and only if (2.17) is true. Therefore, we conclude that (2.17) holds. Now, we know that the power series (2.16) converges in a neighborhood of 0 . Therefore, there exists a positive constant $P$ such that

$$
C_{n}<P^{n}
$$

for $n=1,2,3, \ldots$. In the following lemma, we show that $\left|b_{n}\right| \leq C_{n} d_{n}, n=1,2, \ldots$, where the sequence $\left\{d_{n}\right\}_{n=1}^{\infty}$ is defined as in Lemma 2.1. Indeed, $\left|b_{1}\right|=|\eta| \leq 1=C_{1} d_{1}$, so it suffices to prove that $\left|b_{n+1}\right| \leq C_{n+1} d_{n+1}, n=1,2, \ldots$ Let $P(n)$ denote the statement that $\left|b_{n+1}\right| \leq C_{n+1} d_{n+1}$. From (2.14) and (2.17), we obtain

$$
\begin{aligned}
\left|b_{2}\right| & \leq\left(\sum_{j=0}^{m}\left|c_{j}\right|\right)|\alpha-1|^{-1}\left|b_{0}\right|\left|b_{1}\right|\left|b_{1}\right|\left|b_{0}\right| \\
& \leq M|\alpha-1|^{-1} C_{0} C_{1} d_{1} C_{1} d_{1} C_{0} \\
& =\left(M C_{0} C_{1} C_{1} C_{0}\right)\left(|\alpha-1|^{-1} d_{1} d_{1}\right) \\
& =C_{2}|\alpha-1|^{-1} \max _{\substack{n_{1}+n_{2}=2 \\
0<n_{1} \leq n_{2}}}\left\{d_{n_{1}} d_{n_{2}}\right\}=C_{2} d_{2} .
\end{aligned}
$$

Thus, $P(2)$ is true. Next, suppose that $P(1), P(2), \ldots, P(n)$ are true, that is, $\left|b_{s+1}\right| \leq C_{s+1} d_{s+1}$, for all $s=1,2, \ldots, n$. By (2.14) and (2.17), we get that

$$
\left|b_{n+2}\right| \leq \frac{M}{\left|\left(\alpha^{n+1}-1\right)\right|} \sum_{s=0}^{n} \sum_{k=0}^{n-s} \sum_{i=0}^{n-k-s}\left|b_{i}\right|\left|b_{k+1}\right|\left|b_{s+1}\right|\left|b_{n-s-k-i}\right|
$$




$$
\begin{aligned}
& =\frac{M}{\left|\left(\alpha^{n+1}-1\right)\right|} \sum_{s=0}^{n} \sum_{k=0}^{n-s}\left(\left|b_{0}\right|\left|b_{k+1}\right|\left|b_{s+1}\right|\left|b_{n-s-k}\right|\right. \\
& +\left|b_{n-s-k}\right|\left|b_{k+1}\right|\left|b_{s+1}\right|\left|b_{0}\right| \\
& \left.+\sum_{i=1}^{n-k-s-1}\left|b_{i}\right|\left|b_{k+1}\right|\left|b_{s+1}\right|\left|b_{n-s-k-i}\right|\right) \\
& =\frac{M}{\left|\left(\alpha^{n+1}-1\right)\right|} \sum_{s=0}^{n} \sum_{k=0}^{n-s}\left(2\left|b_{0}\right|\left|b_{k+1}\right|\left|b_{s+1}\right|\left|b_{n-s-k}\right|\right. \\
& \left.+\sum_{i=1}^{n-k-s-1}\left|b_{i}\right|\left|b_{k+1}\right|\left|b_{s+1}\right|\left|b_{n-s-k-i}\right|\right) \\
& =\frac{M}{\left|\left(\alpha^{n+1}-1\right)\right|}\left(\sum_{s=0}^{n} \sum_{k=0}^{n-s} 2\left|b_{0}\right|\left|b_{k+1}\right|\left|b_{s+1}\right|\left|b_{n-s-k}\right|\right. \\
& \left.+\sum_{s=0}^{n} \sum_{k=0}^{n-s} \sum_{i=1}^{n-k-s-1}\left|b_{i}\right|\left|b_{k+1}\right|\left|b_{s+1}\right|\left|b_{n-s-k-i}\right|\right) \\
& =\frac{M}{\left|\left(\alpha^{n+1}-1\right)\right|}\left[\sum _ { s = 0 } ^ { n } \left(2\left|b_{0}\right|\left|b_{n-s+1}\right|\left|b_{s+1}\right|\left|b_{0}\right|\right.\right. \\
& \left.+\sum_{k=0}^{n-s-1} 2\left|b_{0}\right|\left|b_{k+1}\right|\left|b_{s+1}\right|\left|b_{n-s-k}\right|\right) \\
& \left.+\sum_{s=0}^{n} \sum_{k=0}^{n-s} \sum_{i=1}^{n-k-s-1}\left|b_{i}\right|\left|b_{k+1}\right|\left|b_{s+1}\right|\left|b_{n-s-k-i}\right|\right] \\
& =\frac{M}{\left|\left(\alpha^{n+1}-1\right)\right|}\left[\sum_{s=0}^{n} 2\left|b_{0}\right|\left|b_{n-s+1}\right|\left|b_{s+1}\right|\left|b_{0}\right|\right. \\
& +\sum_{s=0}^{n} \sum_{k=0}^{n-s-1} 2\left|b_{0}\right|\left|b_{k+1}\right|\left|b_{s+1}\right|\left|b_{n-s-k}\right| \\
& \left.+\sum_{s=0}^{n} \sum_{k=0}^{n-s} \sum_{i=1}^{n-k-s-1}\left|b_{i}\right|\left|b_{k+1}\right|\left|b_{s+1}\right|\left|b_{n-s-k-i}\right|\right] \\
& \leq \frac{M}{\left|\left(\alpha^{n+1}-1\right)\right|}\left[\sum_{s=0}^{n} 2 C_{0}^{2} C_{n-s+1} d_{n-s+1} C_{s+1} d_{s+1}\right. \\
& +\sum_{s=0}^{n} \sum_{k=0}^{n-s-1} 2 C_{0} C_{k+1} d_{k+1} C_{s+1} d_{s+1} C_{n-s-k} d_{n-s-k} \\
& \left.+\sum_{s=0}^{n} \sum_{k=0}^{n-s} \sum_{i=1}^{n-k-s-1} C_{i} d_{i} C_{k+1} d_{k+1} C_{s+1} d_{s+1} C_{n-s-k-i} d_{n-s-k-i}\right]
\end{aligned}
$$


8 Analytic solution of functional differential equation

$$
\begin{aligned}
&=\frac{M}{\left|\left(\alpha^{n+1}-1\right)\right|}\left[\sum_{s=0}^{n} 2 C_{0}^{2} C_{n-s+1} C_{s+1} d_{n-s+1} d_{s+1}\right. \\
&+\sum_{s=0}^{n} \sum_{k=0}^{n-s-1} 2 C_{0} C_{k+1} C_{s+1} C_{n-s-k} d_{k+1} d_{s+1} d_{n-s-k} \\
&\left.+\sum_{s=0}^{n} \sum_{k=0}^{n-s} \sum_{i=1}^{n-k-s-1} C_{i} C_{k+1} C_{s+1} C_{n-s-k-i} d_{i} d_{k+1} d_{s+1} d_{n-s-k-i}\right] \\
& \leq \frac{M}{\left|\left(\alpha^{n+1}-1\right)\right|} \max _{\substack{n_{1}+n_{2}+\cdots+n_{t}=n+2 \\
0<n_{1} \leq n_{2} \leq \cdots \leq n_{t}, t \geq 2}}\left\{d_{n_{1}} d_{n_{2}} \cdots d_{n_{t}}\right\} \\
& \times\left[\sum_{s=0}^{n} 2 C_{0}^{2} C_{n-s+1} C_{s+1}+\sum_{s=0}^{n} \sum_{k=0}^{n-s-1} 2 C_{0} C_{k+1} C_{s+1} C_{n-s-k}\right. \\
&\left.+\sum_{s=0}^{n} \sum_{k=0}^{n-s} \sum_{i=1}^{n-k-s-1} C_{i} C_{k+1} C_{s+1} C_{n-s-k-i}\right] \\
&= d_{n+2} C_{n+2} .
\end{aligned}
$$

Therefore, $P(n+1)$ is true, we conclude that $\left|b_{n}\right| \leq C_{n} d_{n}$, for all $n=1,2,3, \ldots$ In view of (2.21) and Lemma 2.1, we see that

$$
\left|b_{n}\right| \leq P^{n}\left(2^{5 \delta+1}\right)^{n-1} n^{-2 \delta}, \quad n=1,2,3, \ldots
$$

Therefore,

$$
\begin{aligned}
\limsup \left|b_{n}\right|^{1 / n} & \leq \limsup P\left(2^{5 \delta+1}\right)^{(n-1) / n} n^{-2 \delta / n} \\
& =\lim P\left(2^{5 \delta+1}\right)^{(n-1) / n} n^{-2 \delta / n}=P 2^{5 \delta+1} .
\end{aligned}
$$

Thus, $1 / \limsup \left|b_{n}\right|^{1 / n} \geq 1 / P 2^{5 \delta+1}$, which shows that power series (2.2) converges for $|z|<1 / P 2^{5 \delta+1}$. The proof is complete.

Lemma 2.3. Suppose that (H1) holds. Then for any $r \geq m,(1.3)$ has an analytic solution of the form (2.2) in a neighborhood of 0.

Proof. For $r \geq m, 0 \leq k+s \leq n$, we have $s+1 \leq n+1$, and $k+1 \leq n-s+1$, it follows that $(s+1) /(n+1) \leq 1$ and $(k+1) /(n-s+1) \leq 1$. Next, we have

$$
\begin{aligned}
(k+s+1) r+j(n-s-k)-r n \\
=(k+s) r+r-(k+s) j+j n-r n \\
=(k+s)(r-j)-n(r-j)+r \\
=(k+s-n)(r-j)+r, \quad \text { so } \\
(k+s+1) r+j(n-s-k)-r n=(k+s-n)(r-j)+r .
\end{aligned}
$$


Since $|\alpha|>1,|\alpha|^{(k+s+1) r+j(n-s-k)-r n}=|\alpha|^{(k+s-n)(r-j)+r}=|\alpha|^{(k+s-n)(r-j)}|\alpha|^{r} \leq|\alpha|^{r}$ and the sequence

$$
\left\{\frac{|\alpha|^{r-1} \sum_{j=0}^{m}\left|c_{j}\right|}{|\alpha|^{n+1}-1}\right\}_{n=1}^{\infty}
$$

converges to 0 , this sequence is bounded, namely, there exists $M>0$ such that

$$
\frac{|\alpha|^{r-1} \sum_{j=0}^{m}\left|c_{j}\right|}{|\alpha|^{n+1}-1} \leq M, \quad \forall n=1,2,3, \ldots
$$

Therefore,

$$
\begin{aligned}
& \left|\frac{(s+1)(k+1) \sum_{j=0}^{m}\left|c_{j}\right||\alpha|^{(k+s+1) r+j(n-s-k)-r n-1}}{(n+2)(n-s+1)\left(\alpha^{n+1}-1\right)}\right| \\
& \quad \leq \frac{|\alpha|^{r-1} \sum_{j=0}^{m}\left|c_{j}\right|}{\left|\alpha^{n+1}-1\right|} \leq \frac{|\alpha|^{r-1} \sum_{j=0}^{m}\left|c_{j}\right|}{|\alpha|^{n+1}-1} \leq M, \quad \forall n=1,2,3, \ldots .
\end{aligned}
$$

In view of (2.10), we get that

$$
\left|b_{n+2}\right| \leq M \sum_{s=0}^{n} \sum_{k=0}^{n-s} \sum_{i=0}^{n-k-s}\left|b_{i}\right| b_{s+1}\left|b_{k+1}\right|\left|b_{n-s-k-i}\right|, \quad \forall n=0,1,2, \ldots
$$

We define a sequence $\left\{D_{n}\right\}_{n=0}^{\infty}$ by $D_{0}=|\mu|, D_{1}=|\eta|$ and

$$
D_{n+2}=M \sum_{s=0}^{n} \sum_{k=0}^{n-s} \sum_{i=0}^{n-k-s} D_{i} D_{s+1} D_{k+1} D_{n-s-k-i}, \quad \forall n=0,1,2, \ldots
$$

Next, we will show that $\left|b_{n+1}\right| \leq D_{n+1}, n=1,2,3, \ldots$ By definition of $D_{n}$, we have $\left|b_{0}\right| \leq$ $D_{0},\left|b_{1}\right| \leq D_{1}$ and we let $P(n)$ denote the statement that $\left|b_{n+1}\right| \leq D_{n+1}$. Then

$$
\begin{aligned}
\left|b_{2}\right| & \leq M \sum_{s=0}^{0} \sum_{k=0}^{0-s} \sum_{i=0}^{0-k-s}\left|b_{i}\right| b_{s+1}\left|b_{k+1}\right|\left|b_{0-s-k-i}\right| \\
& =M\left|b_{0}\right|\left|b_{1}\right|\left|b_{1}\right|\left|b_{0}\right|=M\left|b_{0}\right|^{2}\left|b_{1}\right|^{2}=D_{2} .
\end{aligned}
$$

Therefore, $P(1)$ is true. Next, suppose that $P(1), P(2), \ldots, P(n)$ are true, so $\left|b_{t+1}\right| \leq D_{t+1}$, for $t=1,2,3, \ldots, n$. Therefore,

$$
\begin{aligned}
\left|b_{n+2}\right| & \leq M \sum_{s=0}^{n} \sum_{k=0}^{n-s} \sum_{i=0}^{n-k-s}\left|b_{i}\right| b_{s+1}\left|b_{k+1}\right|\left|b_{n-s-k-i}\right| \\
& \leq M \sum_{s=0}^{n} \sum_{k=0}^{n-s} \sum_{i=0}^{n-k-s} D_{i} D_{s+1} D_{k+1} D_{n-s-k-i}=D_{n+2} .
\end{aligned}
$$


Hence, $P(n+1)$ is true, so we can conclude that $\left|b_{n}\right| \leq D_{n}$, for $n=0,1,2, \ldots$. Now, if we define

$$
G(z)=\sum_{n=0}^{+\infty} D_{n} z^{n}
$$

then

$$
\begin{aligned}
G^{3}(z)= & G(z) G^{2}(z)=\left(D_{0}+\sum_{n=0}^{+\infty} D_{n+1} z^{n+1}\right)\left(\sum_{n=0}^{+\infty}\left(\sum_{i=0}^{n} D_{i} D_{n-i}\right) z^{n}\right) \\
= & D_{0} \sum_{n=0}^{+\infty}\left(\sum_{i=0}^{n} D_{i} D_{n-i}\right) z^{n}+\sum_{n=0}^{+\infty}\left(\sum_{k=0}^{n} \sum_{i=0}^{n-k} D_{i} D_{k+1} D_{n-k-i}\right) z^{n+1}, \\
G^{4}(z)= & G(z) G^{3}(z)=\left(D_{0}+\sum_{n=0}^{+\infty} D_{n+1} z^{n+1}\right) \\
& \times\left[D_{0} \sum_{n=0}^{+\infty}\left(\sum_{i=0}^{n} D_{i} D_{n-i}\right) z^{n}+\sum_{n=0}^{+\infty}\left(\sum_{k=0}^{n} \sum_{i=0}^{n-k} D_{i} D_{k+1} D_{n-k-i}\right) z^{n+1}\right] \\
& +\sum_{n=0}^{+\infty}\left(M \sum_{s=0}^{n} \sum_{k=0}^{+\infty}\left(\sum_{i=0}^{n} \sum_{i=0}^{n-s} D_{n-i}\right) z^{n}+2 D_{0} \sum_{n=0}^{+\infty}\left(\sum_{k=0}^{n-k} \sum_{i=0}^{n} D_{i} D_{k+1} D_{n-k-i} D_{k+1} D_{n-s-k-i}\right) z^{n+1}\right. \\
= & D_{0}^{2} G^{2}(z)+2 D_{0}\left[G^{3}(z)-D_{0} G^{2}(z)\right]+\frac{1}{M} \sum_{n=0}^{+\infty} D_{n+2} z^{n+2} \\
= & 2 D_{0} G^{3}(z)-D_{0}^{2} G^{2}(z)+\frac{1}{M}\left(G(z)-D_{0}-D_{1} z\right) \\
= & 2|\mu| G^{3}(z)-|\mu|^{2} G^{2}(z)+\frac{1}{M}(G(z)-|\mu|-|\eta| z) .
\end{aligned}
$$

Thus,

$$
G^{4}(z)-2|\mu| G^{3}(z)+|\mu|^{2} G^{2}(z)-\frac{1}{M}(G(z)-|\mu|-|\eta| z)=0
$$

Let

$$
R(z, \omega)=\omega^{4}-2|\mu| \omega^{3}+|\mu|^{2} \omega^{2}-\frac{1}{M}(\omega-|\mu|-|\eta| z)
$$

for $(z, \omega)$ in a neighborhood of $(0,|\mu|)$, so we see that $R(0, \mu)=|\mu|^{4}-2|\mu|^{4}+|\mu|^{4}-$ $(1 / M)(|\mu|-|\mu|-|\eta| 0)=0$ and $R_{\omega}^{\prime}(z, \omega)=4 \omega^{3}-6|\mu| \omega^{2}+2|\mu|^{2} \omega-1 / M$, then $R_{\omega}^{\prime}(0$, $|\mu|)=-1 / M \neq 0$. Therefore, there exists a unique function $\omega(z)$ which is analytic in a 
neighborhood of 0 such that $\omega(0)=|\mu|, \omega^{\prime}(0)=|\eta|$ and satisfies $R(z, \omega(z))=0$. According to (2.34) and (2.36), we have $G(z)=\omega(z)$. It follows that the power series (2.34) converges in a neighborhood of 0 , which implies that the power series (2.2) is also convergent in a neighborhood of 0 . The proof is complete.

Lemma 2.4. Suppose that (H2) holds. Then for either $0<r \leq m$ and $c_{0}=0, c_{1}=0, \ldots, c_{r-1}=$ 0 , or $r=0,(1.3)$ has an analytic solution of the from (2.2) in a neighborhood of 0.

Proof. By assumption, we get that

$$
\left\{\frac{|\alpha|^{-1} \sum_{j=0}^{m}\left|c_{j}\right|}{1-|\alpha|^{n+1}}\right\}_{n=1}^{+\infty}
$$

converges to $|\alpha|^{-1} \sum_{j=0}^{m}\left|c_{j}\right|$, so it is a bounded sequence which implies that there exists $M>0$ such that

$$
\frac{|\alpha|^{-1} \sum_{j=0}^{m}\left|c_{j}\right|}{1-|\alpha|^{n+1}} \leq M, \quad \forall n=1,2,3, \ldots
$$

There are two cases to consider as follows.

Case 1. $r=0$. As $0 \leq k+s \leq n$, we have

$$
\begin{aligned}
& \left|\frac{(s+1)(k+1) \sum_{j=0}^{m} c_{j} \alpha^{(n-s-k) j+(k+s+1) r-r n-1}}{(n+2)(n-s+1)\left(\alpha^{n+1}-1\right)}\right| \\
& =\left|\frac{(s+1)(k+1) \sum_{j=0}^{m} c_{j} \alpha^{(n-s-k) j-1}}{(n+2)(n-s+1)\left(\alpha^{n+1}-1\right)}\right| \leq \frac{|\alpha|^{-1} \sum_{j=0}^{m}\left|c_{j}\right|}{1-|\alpha|^{n+1}} .
\end{aligned}
$$

Case 2. $0<r \leq m$ and $c_{0}=0, c_{1}=0, \ldots, c_{r-1}=0$. We see that $|\alpha|^{(r-j)(k+s-n)} \leq 1$, where $r \leq j \leq m$. Then,

$$
\begin{aligned}
|\alpha|^{(n-s-k) j+(k+s+1) r-r n-1} & =|\alpha|^{(r-j)(k+s-n)+r-1} \\
& =|\alpha|^{(r-j)(k+s-n)}|\alpha|^{r}|\alpha|^{-1} \leq|\alpha|^{-1} .
\end{aligned}
$$

Thus,

$$
|\alpha|^{(n-s-k) j+(k+s+1) r-r n-1} \leq|\alpha|^{-1} .
$$

Next, we consider

$$
\begin{aligned}
& \left|\frac{(s+1)(k+1) \sum_{j=0}^{m} c_{j} \alpha^{(n-s-k) j+(k+s+1) r-r n-1}}{(n+2)(n-s+1)\left(\alpha^{n+1}-1\right)}\right| \\
& \quad \leq \frac{\sum_{j=0}^{m}\left|c_{j}\right||\alpha|^{(n-s-k) j+(k+s+1) r-r n-1}}{1-|\alpha|^{n+1}} \leq \frac{|\alpha|^{-1} \sum_{j=0}^{m}\left|c_{j}\right|}{1-|\alpha|^{n+1}} .
\end{aligned}
$$


Therefore, by both cases, we have

$$
\left|\frac{(s+1)(k+1) \sum_{j=0}^{m} c_{j} \alpha^{(n-s-k) j+(k+s+1) r-r n-1}}{(n+2)(n-s+1)\left(\alpha^{n+1}-1\right)}\right| \leq \frac{|\alpha|^{-1} \sum_{j=0}^{m}\left|c_{j}\right|}{1-|\alpha|^{n+1}} .
$$

It follows that

$$
\left|\frac{(s+1)(k+1) \sum_{j=0}^{m} c_{j} \alpha^{(n-s-k) j+(k+s+1) r-r n-1}}{(n+2)(n-s+1)\left(\alpha^{n+1}-1\right)}\right| \leq M \quad \text { for } n=1,2,3, \ldots
$$

The conclusion of Lemma 2.4 now follows easily from the same argument as in the proof of Lemma 2.3.

\section{Main results}

We now state the main result of this paper. Consider the following three hypotheses:

(i) (H3) holds;

(ii) (H1) holds, and $r \geq m$;

(iii) (H2) holds, and either $0<r \leq m$ and $c_{0}=0, c_{1}=0, \ldots, c_{r-1}=0$, or $r=0$.

Theorem 3.1. Suppose one of the conditions (i), (ii), or (iii) is fulfilled. Then, for any $\mu$, (1.2) has an analytic solution $x(z)$ in a neighborhood of $\mu$ satisfying the initial conditions $x(\mu)=\mu, x^{\prime}(\mu)=\alpha$. This solution has the form $x(z)=y\left(\alpha y^{-1}(z)\right)$, where $y(z)$ is an analytic solution of the initial value problem (1.3)-(1.4).

Proof. In view of Lemmas 2.2-2.4, we may find a sequence $\left\{b_{n}\right\}_{n=2}^{\infty}$ such that the function $y(z)$ of the form (2.2) is an analytic solution of (1.3) in a neighborhood of 0 . Since $y^{\prime}(0)=$ $\eta \neq 0$, the function $y^{-1}(z)$ is analytic in a neighborhood of the $y(0)=\mu$. If we define $x(z)$ by means of (1.5), then

$$
\begin{aligned}
x^{\prime \prime}\left(x^{[r]}(z)\right) & =x^{\prime \prime}\left(y\left(\alpha^{r} y^{-1}(z)\right)\right) \\
& =\frac{\alpha^{2} y^{\prime \prime}\left(\alpha^{r+1} y^{-1}(z)\right) y^{\prime}\left(\alpha^{r} y^{-1}(z)\right)-\alpha y^{\prime}\left(\alpha^{r+1} y^{-1}(z)\right) y^{\prime \prime}\left(\alpha^{r} y^{-1}(z)\right)}{\left[y^{\prime}\left(\alpha^{r} y^{-1}(z)\right)\right]^{3}} \\
& =\sum_{j=0}^{m} c_{j}\left(y\left(\alpha^{j} y^{-1}(z)\right)\right)^{2}, \quad \text { by }(1.3), \\
& =\sum_{j=0}^{m} c_{j}\left(x^{[j]}(z)\right)^{2}, \quad \text { as required. }
\end{aligned}
$$

The proof is complete.

We now show how to explicitly construct an analytic solution of (1.2). Since $x(z)=$ $y\left(\alpha y^{-1}(z)\right), x(\mu)=y\left(\alpha y^{-1}(\mu)\right)=y(0)=\mu$. By Theorem 3.1, $x(z)$ is an analytic function in a neighborhood of $\mu$. Thus $x(z)$ can be written in a neighborhood of $\mu$ as

$$
x(z)=\mu+x^{\prime}(\mu)(z-\mu)+\frac{x^{\prime \prime}(\mu)(z-\mu)^{2}}{2 !}+\frac{x^{\prime \prime \prime}(\mu)(z-\mu)^{3}}{3 !}+\cdots
$$


Next, we will determine the derivatives $x^{(n)}(\mu), n=1,2, \ldots$ We have $x(z)=y\left(\alpha y^{-1}(z)\right)$, so that $x^{\prime}(z)=\alpha y^{\prime}\left(\alpha y^{-1}(z)\right) / y^{\prime}\left(y^{-1}(z)\right)$. That is, $x^{\prime}(\mu)=\alpha y^{\prime}\left(\alpha y^{-1}(\mu)\right) / y^{\prime}\left(y^{-1}(\mu)\right)=\alpha y^{\prime}(0) /$ $y^{\prime}(0)=\alpha$. Hence $x^{\prime}(\mu)=\alpha$. By means of (1.2), we get that

$$
x^{\prime \prime}(\mu)=x^{\prime \prime}\left(x^{[r]}(\mu)\right)=\sum_{j=0}^{m} c_{j}\left(x^{[j]}(\mu)\right)^{2}=\mu^{2} \sum_{j=0}^{m} c_{j} ;
$$

hence $x^{\prime \prime}(\mu)=\mu^{2} \sum_{j=0}^{m} c_{j}$. Next, we have

$$
\begin{aligned}
\left(x^{\prime \prime}\left(x^{[r]}(z)\right)\right)^{\prime} & =x^{\prime \prime \prime}\left(x^{[r]}(z)\right)\left(x^{[r]}(z)\right)^{\prime} \\
& =x^{\prime \prime \prime}\left(x^{[r]}(z)\right) x^{\prime}\left(x^{[r-1]}(z)\right) x^{\prime}\left(x^{[r-2]}(z)\right) \cdots x^{\prime}(x(z)) x^{\prime}(z) .
\end{aligned}
$$

Therefore, the derivative of $\left(x^{\prime \prime}\left(x^{[r]}(z)\right)\right)$ at $z=\mu$ is

$$
\begin{aligned}
x^{\prime \prime \prime}\left(x^{[r]}(\mu)\right) x^{\prime}\left(x^{[r-1]}(\mu)\right) x^{\prime}\left(x^{[r-2]}(\mu)\right) \cdots x^{\prime}(x(\mu)) x^{\prime}(\mu)=x^{\prime \prime \prime}(\mu)\left[x^{\prime}(\mu)\right]^{r}=x^{\prime \prime \prime}(\mu) \alpha^{r}, \\
\left(\sum_{j=0}^{m} c_{j}\left(x^{[j]}(z)\right)^{2}\right)^{\prime}=\sum_{j=0}^{m} c_{j}\left(\left(x^{[j]}(z)\right)^{2}\right)^{\prime}=2 \sum_{j=0}^{m} c_{j} x^{[j]}(z)\left(x^{[j]}(z)\right)^{\prime} \\
=2 \sum_{j=0}^{m} c_{j} x^{[j]}(z) x^{\prime}\left(x^{[j-1]}(z)\right) x^{\prime}\left(x^{[j-2]}(z)\right) \cdots x^{\prime}(x(z)) x^{\prime}(z) .
\end{aligned}
$$

Hence, the first derivative of $\left(\sum_{j=0}^{m} c_{j}\left(x^{[j]}(z)\right)^{2}\right)$ at $z=\mu$ is $2 \mu \sum_{j=0}^{m} c_{j} \alpha^{j}$. Next, by taking the first derivative of (1.2) at $z=\mu$, we get that

$$
x^{\prime \prime \prime}(\mu) \alpha^{r}=2 \mu \sum_{j=0}^{m} c_{j} \alpha^{j}
$$

Thus,

$$
x^{\prime \prime \prime}(\mu)=2 \mu \sum_{j=0}^{m} c_{j} \alpha^{j-r} .
$$

In general, we can show by induction that

$$
\begin{aligned}
\left(x^{\prime \prime}\left(x^{[r]}(z)\right)\right)^{(n+1)}= & \left(\left(x^{[r]}(z)\right)^{\prime}\right)^{n+1} x^{(n+3)}\left(x^{[r]}(z)\right) \\
& +\sum_{k=1}^{n}\left[P_{k, n+1}\left(\left(x^{[r]}(z)\right)^{\prime},\left(x^{[r]}(z)\right)^{\prime \prime}, \ldots,\left(x^{[r]}(z)\right)^{(n+1)}\right)\right] x^{(k+2)}\left(x^{[r]}(z)\right),
\end{aligned}
$$

for $n=1,2, \ldots$, and

$$
\left(x^{[j]}(z)\right)^{(l)}=Q_{j l}\left(x_{10}(z), \ldots, x_{1, j-1}(z) ; \ldots ; x_{l 0}(z), \ldots, x_{l, j-1}(z)\right),
$$


14 Analytic solution of functional differential equation

respectively, where $x_{i j}(z)=x^{(i)}\left(x^{[j]}(z)\right), P_{j k}$ and $Q_{j l}$ are polynomials with nonnegative coefficients. Next, we have

$$
\begin{aligned}
\left(\sum_{j=0}^{m} c_{j}\left(\left(x^{[j]}(z)\right)^{2}\right)\right)^{(n+1)} & =\sum_{j=0}^{m} c_{j}\left(\left(x^{[j]}(z)\right)^{2}\right)^{(n+1)} \\
& =2 \sum_{j=0}^{m} c_{j}\left(x^{[j]}(z)\right)\left(\left(x^{[j]}(z)\right)^{\prime}\right)^{(n)} \\
& =2 \sum_{j=0}^{m} c_{j}\left(\sum_{k=0}^{n} C_{k}^{n}\left(x^{[j]}(z)\right)^{(k)}\left(x^{[j]}(z)\right)^{(n-k+1)}\right) \\
& =2 \sum_{j=0}^{m} \sum_{k=0}^{n} c_{j} C_{k}^{n}\left(x^{[j]}(z)\right)^{(k)}\left(x^{[j]}(z)\right)^{(n-k+1)}, \quad n=1,2, \ldots
\end{aligned}
$$

For convenience, we denote the following notations:

$$
\beta_{j l}=Q_{j l}\left(x^{\prime}(\mu), \ldots, x^{\prime}(\mu) ; \ldots ; x^{(j)}(\mu), \ldots, x^{(j)}(\mu)\right),
$$

where the number of repeats of $x^{(t)}(\mu)$ is $l$, for $t=1,2, \ldots, j$. Then, we see that $\beta_{l j}=$ $\left(x^{[j]}(\mu)\right)^{(l)}$. By differentiating (1.1) for $n+1$ times at $z=\mu$, we get

$$
\begin{aligned}
& \left(\left(x^{[r]}(\mu)\right)^{\prime}\right)^{n+1} x^{(n+3)}\left(x^{[r]}(\mu)\right) \\
& \quad+\sum_{k=1}^{n}\left[P_{k, n+1}\left(\left(x^{[r]}(\mu)\right)^{\prime},\left(x^{[r]}(\mu)\right)^{\prime \prime}, \ldots,\left(x^{[r]}(\mu)\right)^{(n+1)}\right)\right] x^{(k+2)}\left(x^{[r]}(\mu)\right) \\
& \quad=2 \sum_{j=0}^{m} \sum_{k=0}^{n} c_{j} C_{k}^{n}\left(x^{[j]}(\mu)\right)^{(k)}\left(x^{[j]}(\mu)\right)^{(n-k+1)} .
\end{aligned}
$$

Thus,

$$
\alpha^{r(n+1)} x^{(n+3)}(\mu)+\sum_{k=1}^{n}\left[P_{k, n+1}\left(\beta_{1 r}, \beta_{2 r}, \ldots, \beta_{n+1, r}\right)\right] x^{(k+2)}(\mu)=2 \sum_{j=0}^{m} \sum_{k=0}^{n} c_{j} C_{k}^{n} \beta_{k j} \beta_{n-k+1, j}
$$

This shows that

$$
x^{(n+3)}(\mu)=\frac{2 \sum_{j=0}^{m} \sum_{k=0}^{n} c_{j} C_{k}^{n} \beta_{k j} \beta_{n-k+1, j}-\sum_{k=1}^{n}\left[P_{k, n+1}\left(\beta_{1 r}, \beta_{2 r}, \ldots, \beta_{n+1, r}\right)\right] x^{(k+2)}(\mu)}{\alpha^{r(n+1)}},
$$


where $n=1,2, \ldots$. By means of this formula, it is then easy to write out the explicit form of our solution $x(z)$ as follows:

$$
\begin{aligned}
x(z)= & \mu+\alpha(z-\mu)+\frac{\mu^{2}}{2 !} \sum_{j=0}^{m} c_{j}(z-\mu)^{2}+\frac{2 \mu}{3 !} \sum_{j=0}^{m} c_{j} \alpha^{j-r}(z-\mu)^{3} \\
& +\sum_{n=1}^{+\infty} \frac{1}{(n+3) !} x^{(n+3)}(\mu)(z-\mu)^{n+3} .
\end{aligned}
$$

Example 3.2. The following example shows how to construct an analytic solution by using the previous argument. Consider the following functional equation:

$$
x^{\prime \prime}(x(z))=x^{2}(z)+\left(x^{[2]}(z)\right)^{2} .
$$

This is just (1.2) with the choice of $r=1, m=2, c_{0}=1, c_{1}=1$, and $c_{2}=1$. We can easily see that (3.16) satisfies condition (iii) of Theorem 3.1; hence, for any complex numbers $\mu$ and $\alpha$ such that $0<|\alpha|<1,(3.16)$ has an analytic solution $x(z)$ in a neighborhood of $\mu$ which satisfies $x(\mu)=\mu$ and $x^{\prime}(\mu)=\alpha$. This analytic solution has the form as in (3.2) in case $r=1, m=2, c_{0}=1, c_{1}=1$, and $c_{2}=1$. We already know that $x(\mu)=\mu$ and $x^{\prime}(\mu)=\alpha$. We will find $x^{(n)}(\mu), n \geq 2$. For $n=2$, it follows from (3.16) that

$$
x^{\prime \prime}(\mu)=x^{\prime \prime}(x(\mu))=x^{2}(\mu)+\left(x^{[2]}(\mu)\right)^{2}=2 \mu^{2} .
$$

For $n=3$, it follows from (3.16) that

$$
x^{\prime \prime}(x(z))^{\prime}=\left(x^{2}(z)\right)^{\prime}+\left(\left(x^{[2]}(z)\right)^{2}\right)^{\prime} .
$$

Thus,

$$
\begin{aligned}
x^{\prime \prime \prime}(x(z)) x^{\prime}(z) & =2 x(z) x^{\prime}(z)+2 x^{[2]}(z) x^{\prime}(x(z)) x^{\prime}(z) \\
& =2 x^{\prime}(z)\left[x(z)+x^{[2]}(z) x^{\prime}(x(z))\right] .
\end{aligned}
$$

By putting $z=\mu$, we obtain

$$
x^{\prime \prime \prime}(\mu) \alpha=2 \alpha[\mu+\mu \alpha]
$$

which gives

$$
x^{\prime \prime \prime}(\mu)=2(1+\alpha) \mu .
$$

Similarly, for $n=4$, we obtain

$$
x^{(4)}(\mu)=2\left(1+2 \mu^{3}+\alpha^{2}\right)+\frac{4(1+\mu) \mu^{3}}{\alpha^{2}} .
$$


By continuing this process, we obtain an analytic solution of (3.16) as

$$
\begin{aligned}
x(z)= & \mu+\alpha(z-\mu)+\mu^{2}(z-\mu)^{2}+\frac{(1+\alpha)}{3}(z-\mu)^{3} \\
& +\left(\frac{1+2 \mu^{3}+\alpha^{2}}{12}+\frac{(1+\mu) \mu^{3}}{6 \alpha^{2}}\right)(z-\mu)^{4}+\cdots .
\end{aligned}
$$

\section{Acknowledgments}

The second author is supported by Thailand Research Fund (Grant no. RSA4780012) and Faculty of Science, Chiang Mai University. The authors thank the referees for careful readings and helpful comments and suggestions.

\section{References}

[1] M. Kuczma, Functional Equations in a Single Variable, Monografie Matematyczne, Tom 46, Polish Scientific, Warszawa, 1968.

[2] J.-G. Si and X.-P. Wang, Analytic solutions of a second-order iterative functional differential equation, Journal of Computational and Applied Mathematics 126 (2000), no. 1-2, 277-285.

[3] _ Analytic solutions of a second-order iterative functional differential equation, Computers \& Mathematics with Applications 43 (2002), no. 1-2, 81-90.

[4] J.-G. Si, X.-P. Wang, and S. S. Cheng, Analytic solutions of a functional-differential equation with a state derivative dependent delay, Aequationes Mathematicae 57 (1999), no. 1, 75-86.

[5] J.-G. Si, X.-P. Wang, and W.-N. Zhang, Analytic invariant curves for a planar map, Applied Mathematics Letters 15 (2002), no. 5, 567-573.

[6] J.-G. Si and W. Zhang, Analytic solutions of a nonlinear iterative equation near neutral fixed points and poles, Journal of Mathematical Analysis and Applications 284 (2003), no. 1, 373-388.

[7] _ Analytic solutions of a class of iterative functional differential equations, Journal of Computational and Applied Mathematics 162 (2004), no. 2, 467-481.

[8] _ Analytic solutions of a second-order nonautonomous iterative functional differential equation, Journal of Mathematical Analysis and Applications 306 (2005), no. 2, 398-412.

[9] J.-G. Si, W. Zhang, and G.-H. Kim, Analytic solutions of an iterative functional differential equation, Applied Mathematics and Computation 150 (2004), no. 3, 647-659.

[10] X.-P. Wang and J.-G. Si, Analytic solutions of an iterative functional differential equation, Journal of Mathematical Analysis and Applications 262 (2001), no. 2, 490-498.

[11] B. Xu, W. Zhang, and J.-G. Si, Analytic solutions of an iterative functional differential equation which may violate the Diophantine condition, Journal of Difference Equations and Applications 10 (2004), no. 2, 201-211.

Theeradach Kaewong: Department of Mathematics, Faculty of Science, Chiang Mai University, Chiang Mai 50200, Thailand

E-mail address: theeradach@tsu.ac.th

Piyapong Niamsup: Department of Mathematics, Faculty of Science, Chiang Mai University,

Chiang Mai 50200, Thailand

E-mail address: scipnmsp@chiangmai.ac.th 


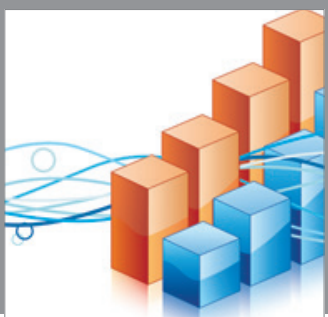

Advances in

Operations Research

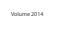

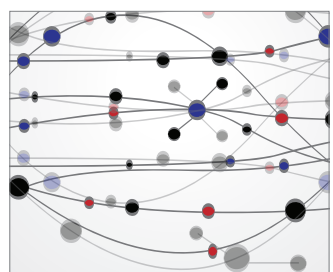

\section{The Scientific} World Journal
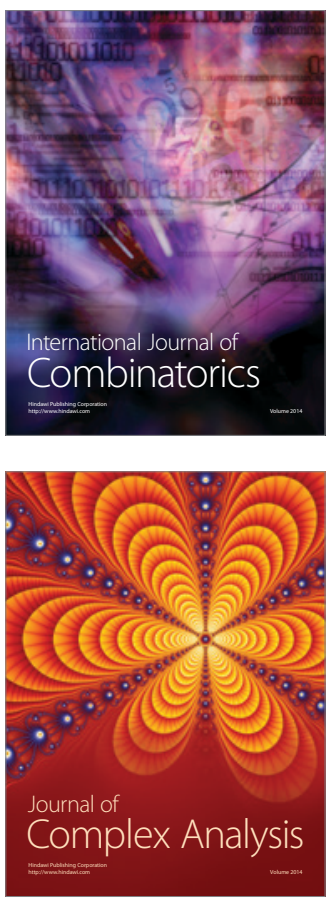

International Journal of

Mathematics and

Mathematical

Sciences
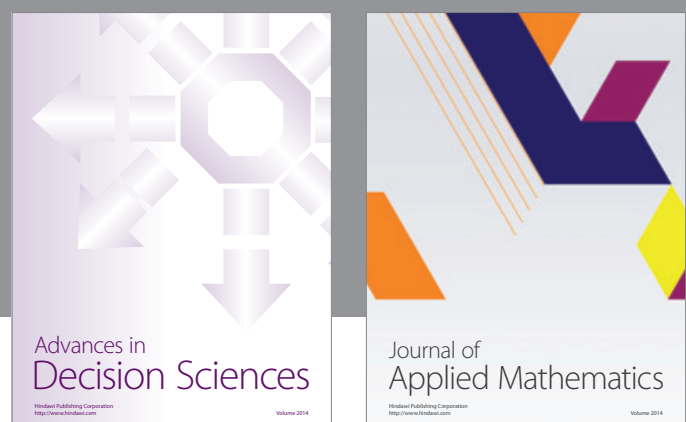

Journal of

Applied Mathematics
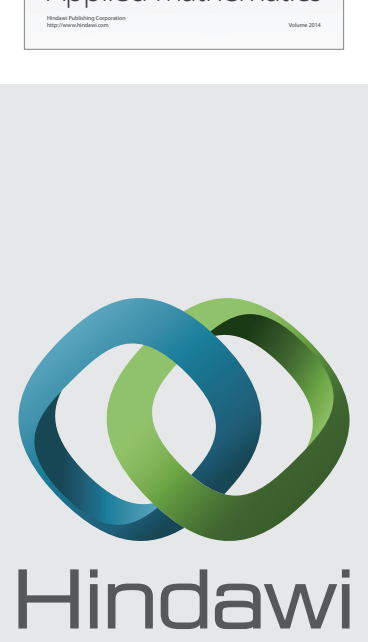

Submit your manuscripts at http://www.hindawi.com
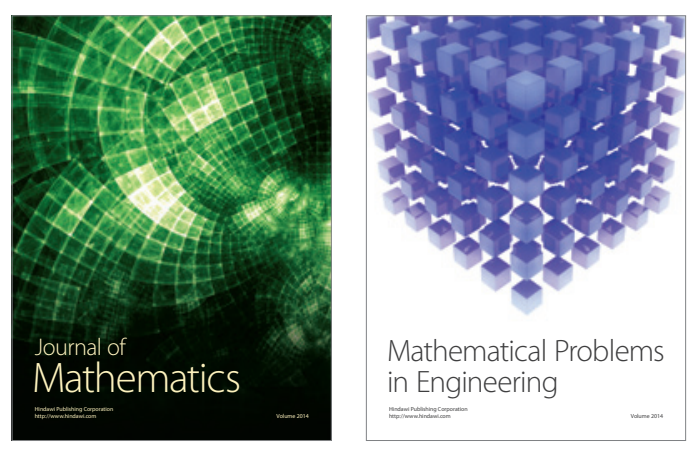

Mathematical Problems in Engineering
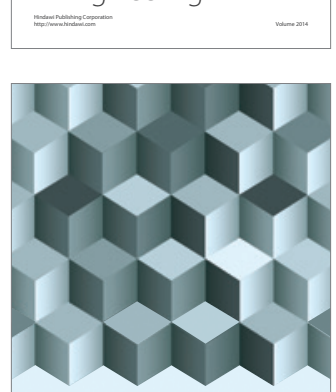

Journal of

Function Spaces
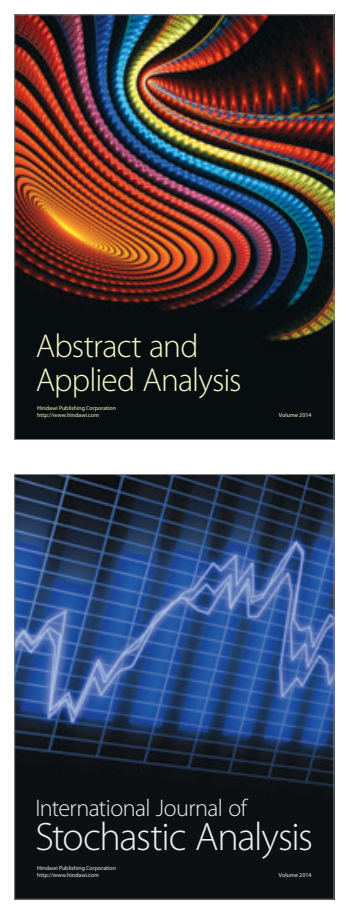

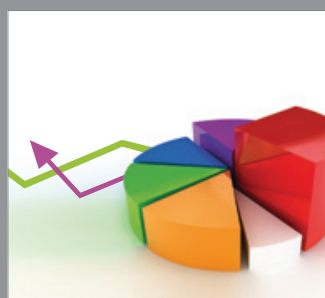

ournal of

Probability and Statistics

Promensencen
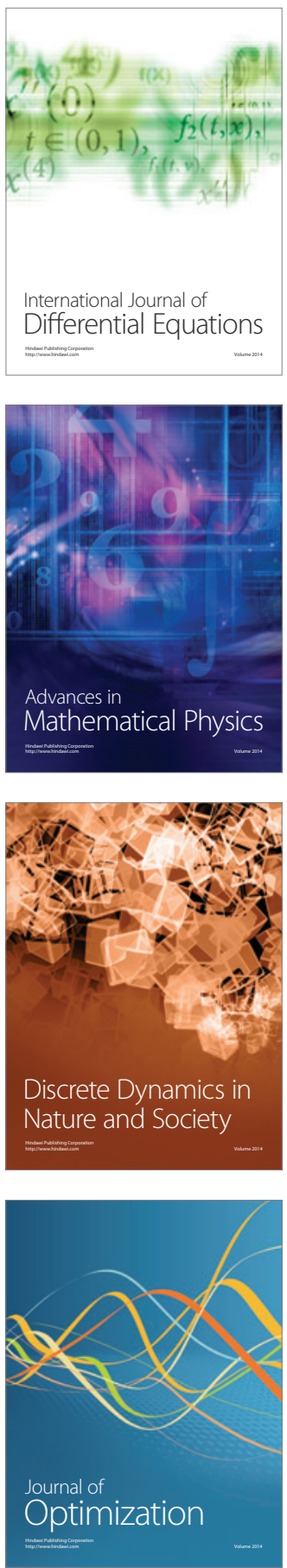